Revue internationale d'éducation de Sèvres

\title{
Amérique latine : résultats contrastés de l'enquête PISA 2009
}

Bernadette Plumelle

\section{OpenEdition}

12 Journals

Édition électronique

URL : https://journals.openedition.org/ries/1023

DOI : $10.4000 /$ ries. 1023

ISSN : 2261-4265

Éditeur

France Education international

Édition imprimée

Date de publication : 1 avril 2011

Pagination : 15-18

ISBN : 978-2854205916

ISSN : $1254-4590$

Référence électronique

Bernadette Plumelle, «Amérique latine : résultats contrastés de l'enquête PISA 2009 », Revue internationale d'éducation de Sèvres [En ligne], 56 | avril 2011, mis en ligne le 01 avril 2014, consulté le 04 mai 2021. URL : http://journals.openedition.org/ries/1023 ; DOI : https://doi.org/10.4000/ries.1023

Ce document a été généré automatiquement le 4 mai 2021.

(c) Tous droits réservés 


\title{
Amérique latine : résultats contrastés de l'enquête PISA 2009
}

\author{
Bernadette Plumelle
}

1 Depuis les résultats de la première enquête en 2000, le Programme international pour le suivi des acquis des élèves (PISA) a suscité et suscite toujours plus d'intérêt dans le public et chez les acteurs de l'éducation. De très nombreux articles ont été produits dans les médias lors de la publication, le 7 décembre 2010, des résultats de l'enquête 2009, et toutes les autorités politiques ont commenté les résultats de leur pays. PISA est désormais une réalité qui s'impose à tous les acteurs de l'éducation : les parents, parce qu'il s'agit d'un classement permettant de «noter» la qualité du système éducatif où sont scolarisés leurs enfants, les chercheurs, qui s'appuient sur la très grande richesse des données comparatives produites par l'enquête pour mener leurs recherches, et les autorités éducatives des pays, qui utilisent les résultats comme levier pour mener des expérimentations et des réformes.

\section{Qu'est-ce que PISA?}

2 L'enquête PISA est avant tout un instrument de suivi : elle évalue tous les trois ans les connaissances et les compétences des élèves en compréhension de l'écrit, en culture mathématique et en culture scientifique, une fois en tant que domaine majeur et deux fois comme domaine mineur, ceci sur une période de neuf ans. Le programme fait passer les mêmes tests, conçus à l'échelon international, aux élèves de 15 ans, en faisant abstraction des différences démographiques et socioculturelles d'un pays à l'autre et sans tenir compte du niveau (classe, filière...) dans lequel ils sont. L'enquête comporte deux volets : une batterie de tests d'évaluation et des questionnaires adressés aux élèves et aux chefs d'établissements.

3 La première évaluation, en 2000, concernait trente-deux pays et portait, comme domaine majeur, sur la compréhension de l'écrit. L'enquête de 2009 porte sur le même domaine, la lecture, ce qui permet pour la première fois d'établir des comparaisons aussi bien dans le temps, avec les performances des élèves évalués il y a une dizaine 
d'années, que dans l'espace, entre pays et plus particulièrement entre pays de la même zone géographique et politique.

\section{Amérique latine, des résultats mitigés}

Des soixante-cinq pays participants, neuf font partie de l'Amérique latine. Le Chili (44e) a obtenu la meilleure position, suivi par l'Uruguay $\left(47^{\mathrm{e}}\right)$, le Mexique $\left(48^{\mathrm{e}}\right)$, la Colombie $\left(52^{\mathrm{e}}\right)$, le Brésil $\left(5^{\mathrm{e}}\right)$, l'Argentine $\left(58^{\mathrm{e}}\right)$, le Panama $\left(62^{\mathrm{e}}\right)$ et le Pérou $\left(63^{\mathrm{e}}\right)$. Les commentaires de la presse de ces pays portent le plus souvent sur les mauvais résultats des pays d'Amérique latine, tous situés dans le bas du tableau à l'échelle mondiale. Cependant, parmi les pays présentant des données comparables entre 2000 et 2009, le Chili, le Pérou et le Brésil ont amélioré leur performance en compréhension de l'écrit.

En quoi se définit cette amélioration? Elle résulte essentiellement d'une amélioration des élèves dans les niveaux les plus faibles (il existe six niveaux, le niveau 1 étant le plus bas), ce qui traduit une progression vers davantage d'équité dans les résultats d'éducation. Le Pérou et l'Argentine ont vu une diminution de la proportion des élèves les moins performants; le Chili présente les résultats les plus spectaculaires, avec une diminution de la proportion des élèves les moins performants de plus de 17 points de pourcentage, et avec une amélioration de la performance des élèves en compréhension de l'écrit, quel que soit le niveau initial.

6 En culture scientifique, le Chili comme le Brésil ont vu diminuer de $5 \%$ le nombre d'élèves sous le niveau 2 de compétences.

7 Ainsi, par comparaison avec l'enquête menée en 2000 sur le même domaine majeur - la compréhension de l'écrit -, les résultats sont meilleurs et les jugements des experts plutôt positifs. Ils doivent être cependant corrigés si l'on regarde les résultats hors comparaison.

8 Au Mexique ou au Chili, la plupart des élèves se classent au niveau 2 de l'échelle de compréhension de l'écrit, le "seuil» de compétence à partir duquel les élèves commencent à montrer qu'ils possèdent des compétences qui leur permettront de participer de manière efficace et productive à la vie de la société. Deuxième caractéristique, au Chili et au Mexique (également au Pérou et en Argentine), les élèves qui fréquentent un établissement urbain affichent de meilleurs scores que les autres élèves, même après contrôle du milieu socio-économique. L'écart est grand : 45 points, soit l'équivalent de plus d'une année d'études, en moyenne. Il est encore plus important au Panama, avec l'équivalent de deux années d'étude.

\section{Des résultats contrastés entre les pays}

En dix ans, les résultats de l'Argentine ont rétrogradé de 418 points à 398 points, alors que les autres pays d'Amérique latine ont connu une hausse comprise entre 16 et 40 points. Peu d'échos ont été donnés aux résultats de PISA dans la presse argentine, qui souligne cependant tout autant les mauvais résultats, comparativement aux autres pays de la zone, que la dégradation de la qualité du système éducatif, alors que la pays était considéré comme à la pointe de l'éducation au $\mathrm{XX}^{\mathrm{e}}$ siècle. Les autorités politiques contestent les résultats. La présidente du gouvernement, Cristina Fernández de Kirchner, considère le test Pisa comme injuste. Selon le ministre de l'éducation, 
Alberto Sileoni, le test PISA a été conçu par les pays riches et ne correspond pas à la réalité de l'Argentine; il a indiqué que l'Argentine pourrait ne plus participer à l'enquête PISA.

Au Chili, les commentateurs soulignent l'amélioration notable des résultats avec une hausse de 39 points par rapport à 2006 et se réjouissent de la première position du pays dans l'ensemble de la zone d'Amérique latine. Cependant, tous dénoncent les très grandes inégalités qui perdurent. Le ministre de l'éducation, Joaquin Lavin, a reconnu qu'au Chili «un élève sur trois n'obtient pas les compétences minimales en compréhension écrite pour bien s'intégrer dans le monde » et, si l'on prend en compte uniquement les élèves de niveau socio-économique faible, $50 \%$ n'acquièrent pas les compétences de base. Les inégalités sont très grandes entre les différents réseaux d'éducation, écoles privées et écoles privées subventionnées, ainsi qu'écoles municipales publiques. Les premières obtiennent une moyenne de 540 points, les écoles privées subventionnées 458 points et les écoles publiques 421 points. Si l'on considère que 40 points de différence sont l'équivalent d'une année de scolarité, on voit qu'il existe un écart de trois ans entre un élève du secteur public et un élève du secteur privé.

11 Au Brésil, les résultats en moyenne ont évolué de 33 points entre 2000 et 2009, mais malgré cette amélioration, le Brésil occupe toujours la $53^{\mathrm{e}}$ place du classement, avant l'Argentine, le Panama et le Pérou, mais derrière le Chili, l'Uruguay, le Mexique et la Colombie. Le « rendement » des élèves des écoles publiques (387 points) est inférieur à celui des élèves des établissements privés (502 points). Les résultats les meilleurs ont été obtenus en mathématiques, où ils sont passés de 334 points en 2000 à 386 points en 2009, de 375 à 405 en sciences et de 396 à 412 en compréhension écrite. L'objectif du Plan d'éducation au développement (2007) était d'atteindre au moins 395 points en moyenne dans les trois matières; le pays a donc atteint ses objectifs. Mais cette place ne satisfait pas le gouvernement pour autant, des pays ayant le même niveau socioéconomique obtenant de meilleurs résultats.

Dans un pays comme le Brésil, où les politiques éducatives sont très décentralisées, les résultats sont très différents selon les États et les écarts importants entre le niveau le plus élevé obtenu par le District fédéral (439) et celui d'un État comme le Maranhao (355), par exemple. Enfin, même si des progrès ont été réalisés, il reste encore plus de $50 \%$ des élèves de 15 ans aux niveaux 1 et 2 de compétence en lecture, sur l'échelle de six niveaux. Pour le ministre de l'Éducation, Fernando Haddad, la revalorisation des salaires des enseignants et leur formation est l'une des deux priorités d'action dans les années à venir. Aucun pays situé dans le groupe des pays ayant les meilleurs résultats ne paie les enseignants à un salaire inférieur à la moyenne des autres professions de l'enseignement supérieur. Or, au Brésil, le salaire des enseignants est de $40 \%$ inférieur aux professions exigeant un diplôme de l'enseignement supérieur.

13 Le ministre a également souligné la nécessité d'un élargissement de l'éducation préscolaire. Une proposition d'amendement à la Constitution, approuvée cette année, exige que d'ici 2016 tous les enfants soient inscrits à l'école dès l'âge de 4 ans alors que l'école est obligatoire actuellement à partir de 6 ans. Des études montrent que le préscolaire a un impact important sur les résultats des élèves à l'école primaire, d'autant plus élevé qu'il touche les populations les plus pauvres, en particulier dans le Nordeste. 


\section{Objectifs de l'éducation pour l'Amérique latine}

14 La lecture de la presse nationale des pays d'Amérique latine montre le très grand décalage entre la perception publique, souvent pessimiste sur l'état du système éducatif des pays, et l'analyse des experts en éducation, qui comparent les résultats 2009 avec ceux de PISA 2000. Mais toutes les réformes envisagées s'orientent vers les mêmes objectifs : réduire les inégalités à l'intérieur du pays, renforcer la formation des enseignants.

Les exemples de réussites ailleurs, comme à Shanghai (Chine), en Pologne et au Qatar jouent comme des signes positifs et les efforts déployés partout montrent que l'amélioration de la qualité de l'éducation est un défi mondial. Les décisions prises dans le cadre du vingtième sommet latino-américain, qui a eu lieu à Mar del Plata en Argentine les 3 et 4 décembre 2010, sont symptomatiques de cette volonté des pays d'Amérique latine d'améliorer la qualité de leur éducation. L'Inter-American Development Bank (IDB) a signé avec les ministres ibéro-américains de l'éducation un accord de coopération qui vise à faire en sorte que soient atteints les objectifs éducatifs en 2021. Les trois domaines stratégiques identifiés par la BID afin d'atteindre une éducation de qualité sont en phase avec les constats qui émergent de l'enquête PISA. Il faut : a) aider au développement de l'éducation préscolaire pour tous les enfants de 0 à 6 ans ; b) soutenir l'amélioration de la qualité de l'enseignement et renforcer la formation des enseignants ; c) améliorer les compétences acquises par les jeunes dans le système éducatif, pour faciliter la transition de l'école à l'emploi.

\section{INDEX}

Index géographique : Amérique latine

Mots-clés : évaluation, PISA : programme international pour le suivi des acquis des élèves, système scolaire

Palabras claves : evaluación, PISA: programa para la evaluación internacional de los alumnos, sistema escolar

Keywords : evaluation, PISA: Program for International Student Assessment, school system

\section{AUTEUR}

BERNADETTE PLUMELLE

Bernadette Plumelle est responsable du centre de ressources et d'ingénierie documentaires du CIEP. 